\title{
Increased miR-188-3p in Ovarian Granulosa Cells of Patients with Polycystic Ovary Syndrome
}

\author{
Beibei Dai $\mathbb{D}^{1}$ and Jun Jiang ${ }^{2}$ \\ ${ }^{1}$ Department of Ultrasound, Obstetrics and Gynecology Hospital of Fudan University, 419 Fangxie Road, Shanghai 200011, China \\ ${ }^{2}$ State Key Laboratory of Genetic Engineering, School of Life Sciences, Fudan University, Shanghai 200438, China \\ Correspondence should be addressed to Beibei Dai; daibeibei0823@126.com
}

Received 19 January 2021; Revised 4 March 2021; Accepted 17 March 2021; Published 15 April 2021

Academic Editor: Tao Huang

Copyright (C) 2021 Beibei Dai and Jun Jiang. This is an open access article distributed under the Creative Commons Attribution License, which permits unrestricted use, distribution, and reproduction in any medium, provided the original work is properly cited.

\begin{abstract}
MicroRNA-target networks are often dysregulated in diseases. Our purpose is to investigate this dysregulation of polycystic ovary syndrome (PCOS). Through the bioinformatics reanalysis of the public RNAseq dataset, we found that miR-188-3p was the miRNA with the highest induction rate, and indicated that miR-188-3p might have a rare function of upregulating its targeted expression. This discovery will increase our understanding of the pathology of PCOS and provide new targets for treatment strategies.
\end{abstract}

\section{Introduction}

Polycystic ovarian syndrome (PCOS) is a hormonal disease common in women of reproductive age [1]. The prevalence of PCOS, following the Rotterdam diagnostic criteria, in a meta-analysis of 15 studies is 10\% [2]. Women with PCOS may have fewer or longer menstrual periods or have highlevel male hormones (androgens), resulting in the ovaries accumulating many small amounts of fluid (follicles) and not releasing eggs regularly [3]. Signs and symptoms of PCOS usually develop around the first monthly period of puberty but can also appear after puberty as the gain of weight. The signs and symptoms of PCOS vary, such as irregular menstrual cycles, excessive androgens, and polycystic ovary. Complications of PCOS include infertility, gestational diabetes, miscarriage, premature birth, metabolic syndrome, abnormal uterine bleeding, and endometrial cancer and bring serious distress to the majority of women $[4,5]$. PCOS is caused by many factors, including excessive insulin, low inflammation, heredity, and excessive androgens. However, it is not clear what is the most important pathogenic factor, which brings great difficulties to clinical treatment [6-8].

Although the pathological mechanism of PCOS has been clarified, the effect of miRNAs on the proliferation of ovarian granulosa cells remains to be further studied [4]. MiRNAs are short, noncoding RNAs that contain approximately 22 nucleotides $[9,10]$. They can regulate messenger RNA (mRNA) expression by binding to complementary nucleotides on the $3^{\prime}$-UTR of the target mRNA. MiRNAs affect the physiological and pathological states of cells by regulating various cellular processes such as cell proliferation, cell differentiation and apoptosis, biosynthesis, and hormone secretion. Pioneers have studied how miRNAs regulate cells and influence the corresponding pathology. For example, recent evidence shows that miR-21, miR27b, miR-103, and miR-155, which are related to human obesity, have clear upregulation in women [11]. Besides, in mouse PCOS models, the enhancement of miR21 and miR-146a is demonstrated to have a potential for DNA damage [12]. Previous studies have also performed miR-222 in membrane cells and found that miR-222 expression can be inhibited by androgens and targeted by cyclindependent kinase inhibitor 1B (p27Kip1), in order to regulate cell proliferation [13]. Subsequent studies on mir-222 have shown that it can reduce estrogen receptor alpha $(\mathrm{ER} \alpha)$ protein levels, related signaling pathways, and targeted gene expression. However, miRNA implication in PCOS is still far from understood. In this bioinformatics study, we investigate miRNAs and their targets dysregulated in PCOS through the 
reanalysis of public RNAseq datasets. This study will bring more insights into the pathogenesis of PCOS and potentially provide new strategies for its therapy.

\section{Materials and Methods}

2.1. Data Resource. GEO DataSets were downloaded from https://www.ncbi.nlm.nih.gov/geo/ via accession numbers GSE138572 and GSE138518.

2.2. Differential Expression miRNA Analysis. Raw read counts were extracted from GEO DataSets. The miRNAs were preexcluded if their maximal read counts among all samples were no more than 5. Batch effect was corrected to make sure the samples with largely variable total read are comparable, using the correction method ComBat and the normalization method TMM. After the batch effect was corrected, PCOS samples were compared with control samples using DESeq2 [14] with the mean fit type and LRT test type via DEBrowser [15].

2.3. Differential Expression $m R N A$ Analysis. Raw read counts were extracted from GEO DataSets. The mRNAs were preexcluded if their maximal read counts among all samples are no more than 100. PCOS samples were compared with control samples using DESeq2 [14] with the mean fit type and LRT test type via DEBrowser [15].

2.4. Collection of Predicted and Validated miRNA Targets. Targets of miRNA were bioinformatically predicted using miRDB (http://mirdb.org), TargetScan (http://www.targetscan .org/vert_72/), and DIANA (http://www.microrna.gr/microTCDS). Experimentally validated miRNA-target interactions were collected from miRTarBase (http://miRTarBase.cuhk.edu.cn/).

2.5. Gene Set Enrichment Analysis (GSEA). Predicted or validated miRNA targets by various algorithms were used to create gmx file. All mRNAs ranked by differential expression statistic were used to create rnk file. The miRNA targets were analyzed to test whether they were enriched in differentially expressed genes using GSEA software [16].

2.6. Overrepresentation Analysis. The selected gene list was analyzed for their overrepresentation in pathways of various databases and gene sets of many published datasets using Enrichr (http://amp.pharm.mssm.edu/Enrichr).

\section{Results}

3.1. miR-188-3p Was Upregulated in PCOS Tissues. To survey the miRNome dysregulation in PCOS, we extracted raw read count data from a publicly available dataset (GSE138572) which included ovarian granulosa cells isolated from five PCOS patients and five healthy controls. Two samples (one from the PCOS group and the other from the controls) had $14.4 \mathrm{M}$ and $6.1 \mathrm{M}$ total annotated reads, respectively, which were much higher than other samples $(2.4 \mathrm{M}$ reads on This read count bias created a strong variance that cannot be adjusted by normalization. Therefore, we treated these two samples as the batch effect, which was corrected using the SVA's ComBat algorithm [17]. After correction, PCOS samples could be separated from controls based on miRNome using the principal component analysis (PCA; Figure 1(a)), with one minor exception. Next, we used DESeq2 via DEBrowser to analyze the fold change (FC) and the adjusted significance $\left(P_{\text {adj }}\right)$ of each miRNA between PCOS and controls. As shown in the volcano plot (Figure 1(b)), most miRNAs had a nonsignificant change in granulosa cells of PCOS (grey dots), except that miR-188-3p was significantly upregulated in PCOS (the red dot). This miRNA was barely expressed in granulosa cells of healthy women but induced in those of PCOS patients (Figure 1(c)).

3.2. Predicted Targets of miR-188-3p Were Upregulated in PCOS Tissues. Since miR-188-3p was a unique miRNA that showed significant dysregulation in ovarian granulosa cells of PCOS patients, we hypothesized that its mRNA targets might be downregulated in PCOS. We surveyed the predicted targets of miR-188-3p using three commonly used algorithms: miRDB [18], TargetScan [19], and DIANA [20]. Then, we used the target lists to create the gmx file for GSEA. In the meantime, we extracted raw read count data from a publicly available mRNAseq dataset (GSE138518) which included ovarian granulosa cells isolated from three PCOS patients and three healthy controls, and used DESeq2 via DEBrowser to analyze the statistic representing the confidence of fold change. The genes with their ranked statistic were used to create the rnk file for GSEA. Both the gmx file and the rnk file were input to GSEA software to evaluate how miR-188-3p target genes were distributed within the ranked differentially expressed genes. Surprisingly, miR-188$3 p$ target genes predicted by either miRDB (Figure 2(a)) or DIANA (Figure 2(b)) were more frequent at top of the ranked list, indicating that they are generally upregulated. The target list predicted by TargetScan was excluded by the GSEA algorithm itself. These data suggest that miR-188-3p functions as an uncommon expression activator, rather than a canonical expression inhibitor, in ovarian granulosa cells.

3.3. Validated Targets of $m i R-188-3 p$ Were Upregulated in PCOS Tissues. To further confirm our unexpected observation, we expanded GSEA analysis to experimentally validated miR-188-3p targets, which were surveyed from the miRTarBase [21]. The full gene list, as well as its subset (genes validated by HITS-CLIP or PAR-CLIP), was used to create the gmx files. Similar to the predicted targets, experimentally validated miR-188-3p targets were also enriched in the top of the ranked differentially expressed genes, based on the whole validated target list (Figure 3(a)), HITS-CLIP validated list (Figure 3(b)), or PAR-CLIP validated list (Figure 3(c)). Gene lists validated by other experimental approaches were excluded from the GSEA analysis due to their limited gene numbers. These data again support the finding that miR188-3p upregulates the expression of targets.

3.4. Upregulated miR-188-3p Targets Were Enriched in PCOS Pathways. To explore the potential functions of genes upregulated by miR-188-3p in ovarian granulosa cells, we selected the targets that contributed to the core enrichment from the full experimentally validated targets via miRTarBase. Next, we performed the classical pathway analysis of this gene 


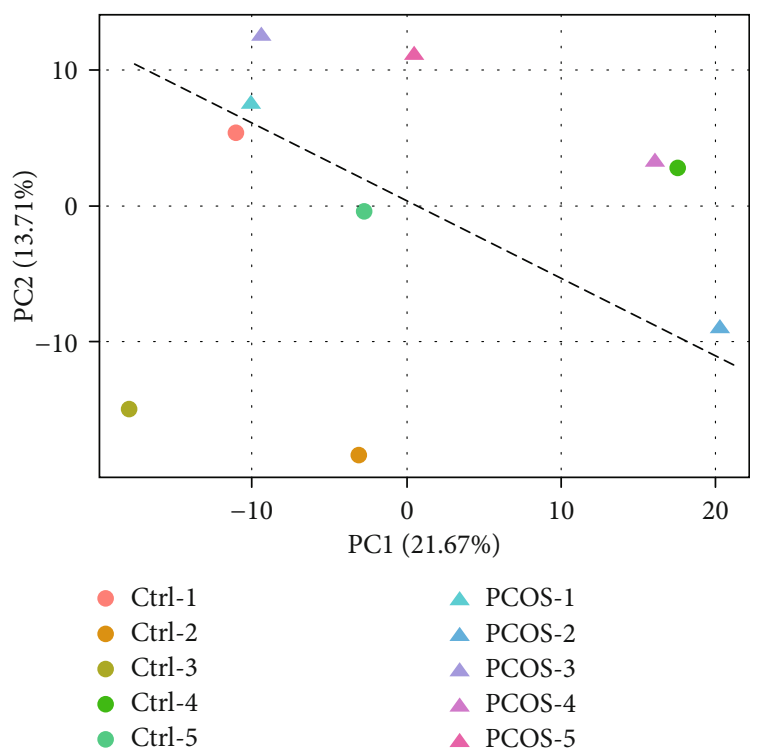

(a)
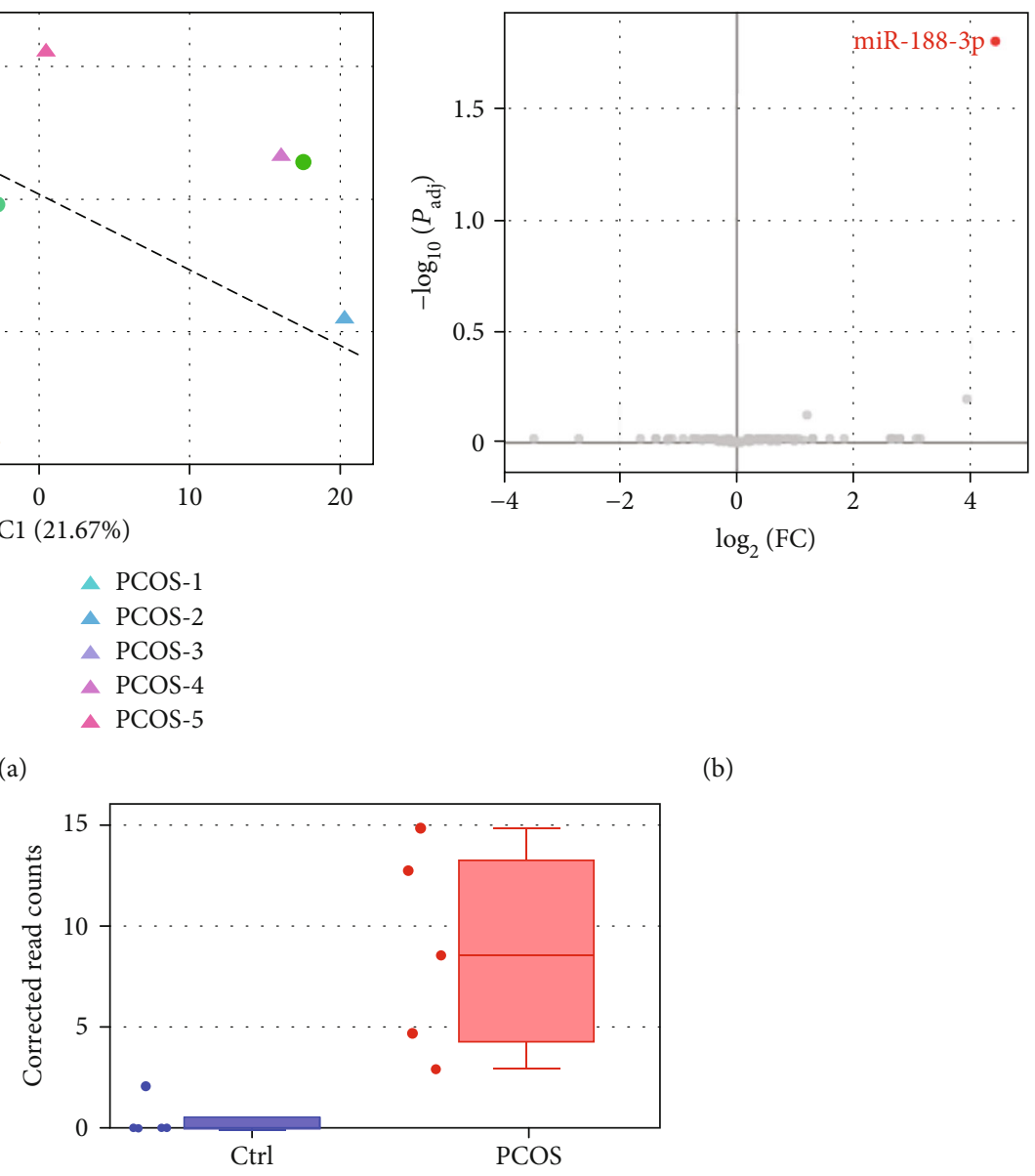

(b)

(c)

FIGURE 1: miR-188-3p was upregulated in PCOS: (a) PCA analysis based on miRNome; (b) volcano plot screening for miRNAs with large fold change $(\mathrm{FC})$ and strong significance $\left(P_{\text {adj }}\right) ;(\mathrm{c})$ corrected read counts of miR-188-3p between PCOS and controls.

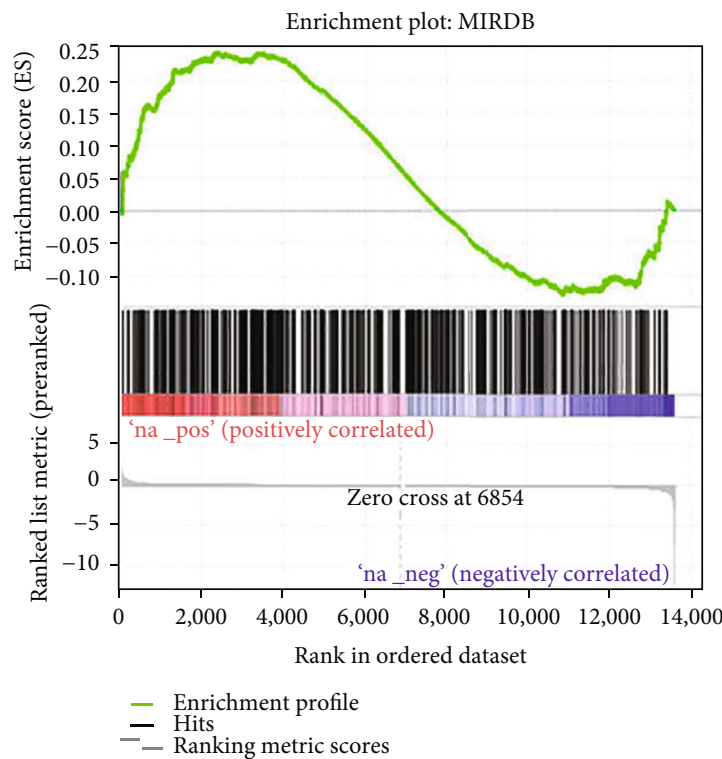

(a)

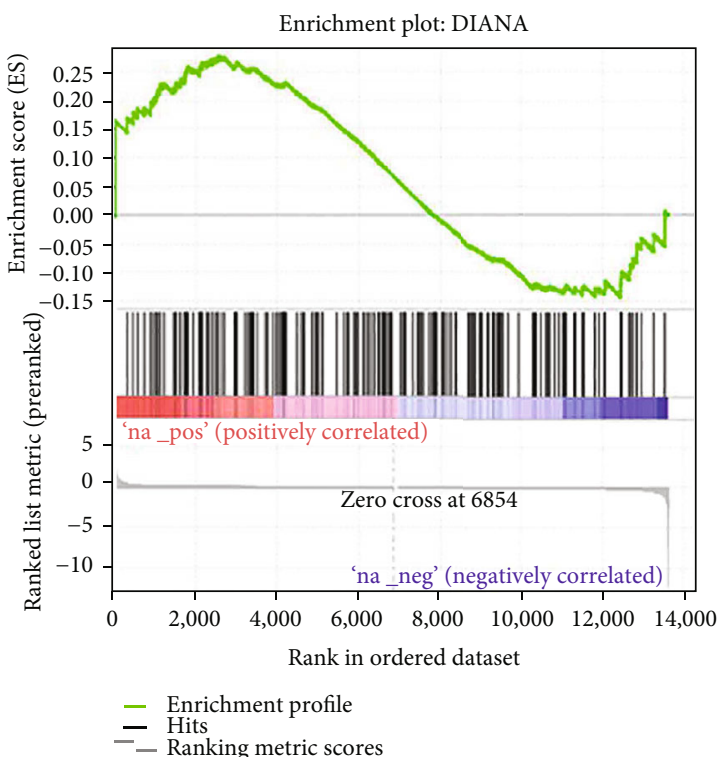

(b)

Figure 2: Predicted targets of miR-188-3p were upregulated in PCOS: (a) miR-188-3p targets predicted by miRDB; (b) miR-188-3p targets predicted by DIANA. 


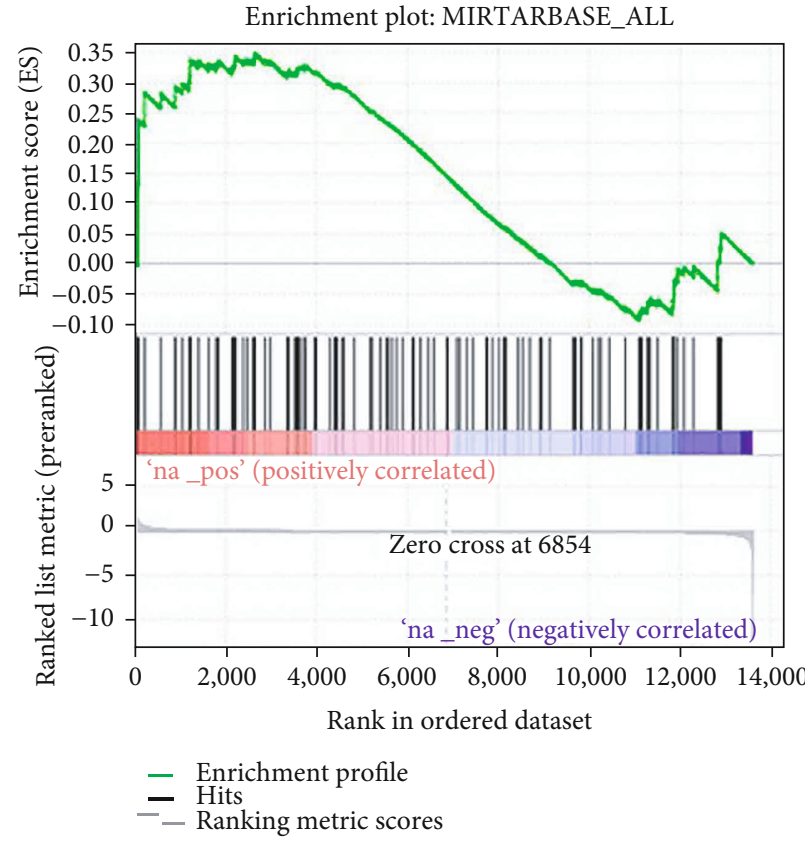

(a)

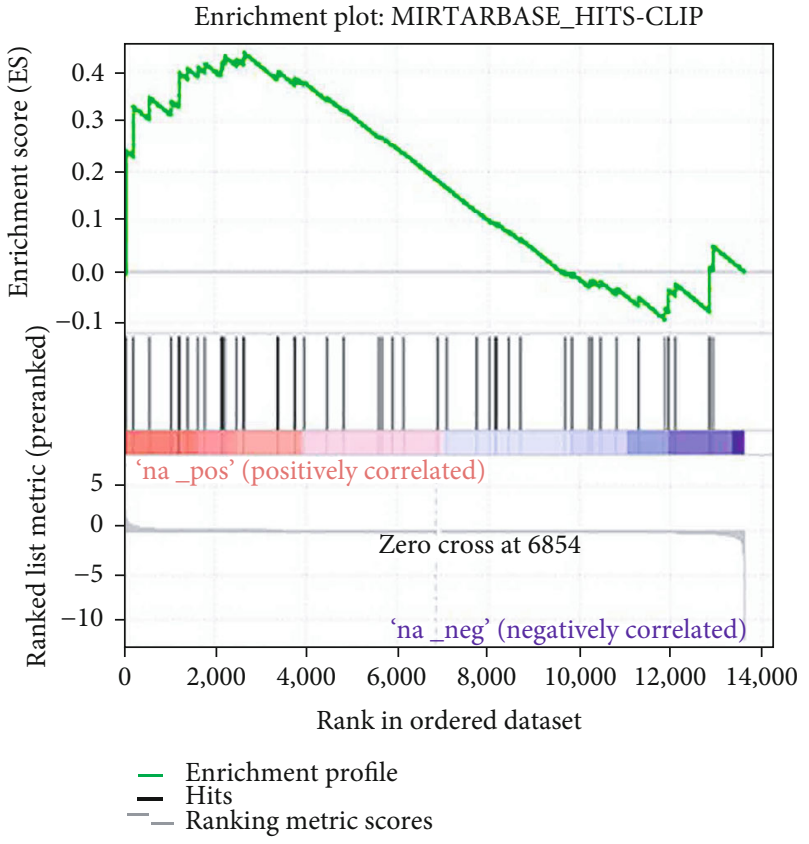

(b)

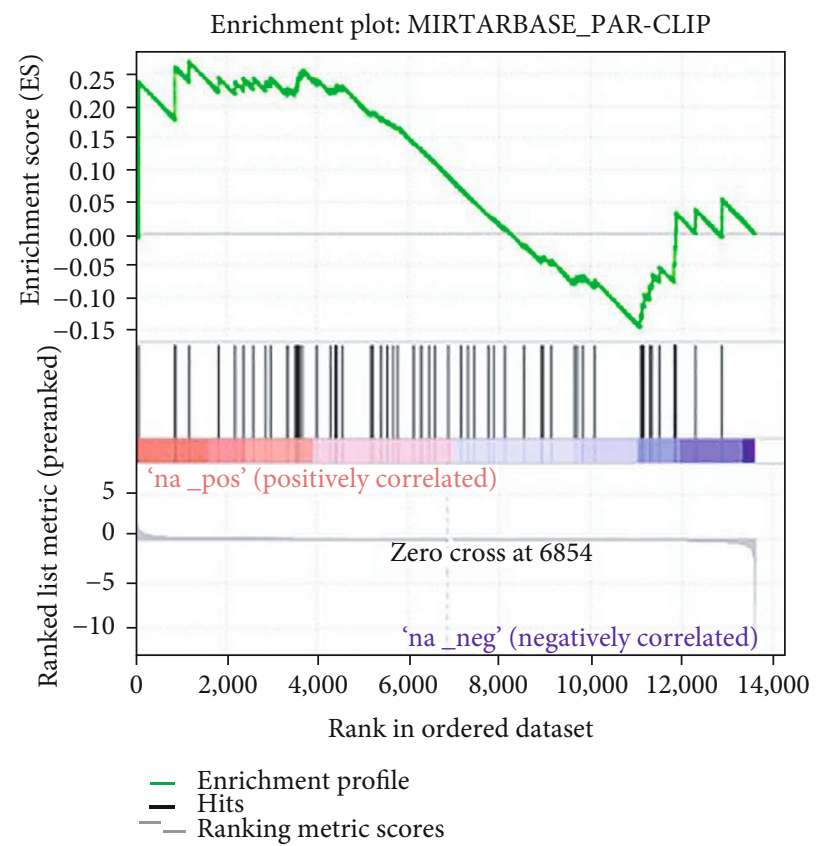

(c)

FIGURE 3: Validated targets of miR-188-3p were upregulated in PCOS: (a) all experimentally validated miR-188-3p targets collected in miRTarBase; (b) miR-188-3p targets validated by HITS-CLIP; (c) miR-188-3p targets validated by PAR-CLIP.

subset using Enrichr [22]. First, we confirmed that these genes are involved in PCOS pathology, as they were also overrepresented in the genes upregulated in PCOS endometrium (GSE48301) [23] from the disease perturbation database (Figure 4(a)). Second, these genes were responding to estradiol (Figure 4(b)) [24] [25], which was consistent with the fact that hormone imbalance is an important aspect of PCOS. Third, the upregulated miR-188-3p targets were enriched in adipogenesis (Figure 4(c)) using WikiPathways database [26], suggesting their implication in weight gain, a common symptom in PCOS patients. And last, these target genes were enriched in the canonical NF- $\kappa \mathrm{B}$ pathways (Figure 4(d)) using the NCI-Nature database [27], providing insights into the potential molecular mechanism of function.

\section{Discussion}

Several miRNAs have been reported to be dysregulated in PCOS ovaries [28]. miR-9 and miR-18b are found to be upregulated in PCOS and change the release of testosterone, 
Disease_Perturbations_from_GEO_up

Inclusion body myositis DOID-3429 human GSE48280 sample 706

Polycystic ovary syndrome DOID-11612 human GSE48301 sample 806

Monoclonal gammopathy of uncertain significance DOID-7442 human GSE47552 sample 563

Sendai virus infection C1319860 mouse GSE10211 sample 82

Cystic Fibrosis C0010674 mouse GSE769 sample 428

Nemaline myopathy DOID-3191 mouse GSE3384 sample 975

Diamond-Blackfan anaemia DOID-1339 human GSE14335 sample 472

Facioscapulohumeral muscular dystrophy DOID-11727 human GSE36398 sample 673

Nemaline myopathy DOID-3191 mouse GSE3384 sample 970

Huntington's disease DOID-12858 human GSE8762 sample 930

(a)

Ligand_Perturbations_from_GEO_up

Interleukin-1 beta mouse embryonic fibroblasts from IRAK-4 kinase deficient mutants GDS2641 ligand:59

Interleukin 1, beta human amnion mesenchymal cells (AMC) GDS4595 ligand:24

TGF-beta mouse embryonic fibroblasts GDS4898 ligand:244

17beta-estradiol mouse bone marrow (BM) c-kit+ stem cells (SCs) GDS3816 ligand:28

Interleukin-1 beta mouse embryonic fibroblasts GDS2641 ligand:57

Interleukin 1, beta human amnion mesenchymal cells (AMC) GDS4595 ligand:23

Thyroid hormone human endometrial cells GDS4987 ligand:168

Growth hormone mouse adipocytes GDS1489 ligand:171

Interleukin-1 beta mouse embryonic fibroblasts GDS2641 ligand:58

Growth hormone mouse 3T3-F442A adipocytes GDS1489 ligand:11

(b)

WikiPathways_2019_Human

Adipogenesis WP236
Small ligand GPCRs WP247
GPCRs, class A rhodopsin-like WP455
Integrated cancer pathway WP1971
Mitochondrial complex I assembly mo
Oxidative phosphorylation WP623
RIG-I-like receptor signaling WP3865
Genotoxicity pathway WP4286
Peptide GPCRs WP24
Human Complement system WP2806

NCI-Nature 2016

Canonical NF-kappaB pathway Homo sapiens 7a1a9c3c-618f-11e5-8ac5-06603eb7f303

Urokinase-type plasminogen activator (uPA) and uPAR-mediated signaling Homo sapiens 503076a2-6196-11e5-8ac5-06603eb7f303

TNF receptor signaling pathway Homo sapiens 316be05e-6196-11e5-8ac5-06603eb7f303

Direct p53 effectors Homo sapiens 67c3b75d-6191-11e5-8ac5-06603eb7f303

(d)

FIGURE 4: Pathway analysis of the validated miR-188-3p targets contributing to core enrichment of GSEA. Overrepresentation analysis was performed using the Enrichr against disease perturbation database (a), ligand perturbation database (b), human WikiPathways database (c), and NCI-Nature database (d). Color code represents the $P$ value. 
progesterone, and estradiol via targeting IL8, SYT1, and IRS2 $[29,30]$. Upregulation of miR-93 in adipose tissue of PCOS patients is found to alter hormone metabolism by targeting GLLU4 [31]. Dysregulation of miR-320 is found to affect estradiol production and release through the regulation of $\mathrm{RAB} 5 \mathrm{~B}$, E2F1, and SF-1 expression $[32,33]$. And the expression of miR-483-5p is affected in PCOS to change granulose cell proliferation by targeting Notch3, MAPK3, and IGF1 [34, 35]. However, these miRNAs show no significant difference in the cohort we analyze, likely due to the limited sample size.

Previously, miR-188-3p has been reported to be involved in various physiologic and pathological processes. Upregulation of $\mathrm{miR}-188-3 \mathrm{p}$ is reported to strongly associate with poor prognosis and short survival in two cohorts of colorectal cancer and may promote cancer cell migration by direct interaction with MLLT4 [36]. Downregulation of miRNA$188-3 p$ is found to contribute to apoptosis of spermatogenic cells in patients with azoospermia by its regulatory role of MLH1 expression [37]. A study of cardiovascular diseases reveals that miR-188-3p suppresses autophagy and myocardial infarction by targeting ATG7 [38]. miR-188-3p is also found to have an inhibitory effect on macrophage inflammatory response and oxidation [39]. Nevertheless, the current study for the first time implicates miR-188-3p in PCOS.

The canonical function of miRNAs is to downregulate the expression of target mRNA, either via mRNA degradation or translational inhibition. However, some miRNAs have been found to upregulate targets in specific cells [10]. The translation activation function of miRNA was first discovered by Vasudevan et al. in the context of the cell cycle [40]. Later, liver-specific miR-122 was shown to activate hepatitis $C$ virus (HCV) RNA expression through recruiting factors involved in RNA replication and translation, as well as increasing RNA stability [41-43]. The wildly expressed miR-10a was reported to interact with ribosomal protein mRNAs and enhance their translation, resulting in the elevation of global protein synthesis and thus cell transformation [44]. Our results in the current study suggest that miR-188-3p performs its regulatory function in such an uncommon way of upregulation in human ovarian granulosa cells, which warrants experimental validation in further studies.

In PCOS, miR-188-3p was found to regulate adipogenesis, which was frequently observed in patients. Approximately $50 \%$ of PCOS patients are overweight or obese [45]. Obesity increases insulin resistance and compensatory hyperinsulinemia, which in turn increases adipogenesis and decreases lipolysis. Obesity also induces insulin resistance and compensatory hyperinsulinemia, glucose intolerance, and dyslipidemia and increases the risk of pregnancy complications [46]. PCOS women with obesity have more severe phenotypes than the ones without obesity, including worse menstrual irregularity, infertility, miscarriage, prematurity, pregnancy-induced hypertension, gestational diabetes, biochemical and clinical hyperandrogenism, glucose intolerance and/or T2DM, and metabolic syndrome [47]. Regulated targets of miR-188-3p in PCOS were also enriched in the NF- $\kappa$ B pathway, suggesting chronic inflammation involved $[48,49]$. Consistently, the phosphatidylinositol 3 -kinase/AKT/NF- $\kappa$ B signaling pathway was reported to mediate WNT5a-induced inflammation and oxidative stress in ovar- ian granulosa cells in PCOS [50]. Furthermore, iridoids (a NF$\kappa \mathrm{B}$ inhibitor) with genipin stem nucleus were proposed to protect PCOS patients from inflammatory damage [51]. All these pathway analyses could guide the further experimental investigation on the molecular mechanism of miR-188-3p implication in PCOS.

In summary, we reanalyzed miRNAseq and mRNAseq data from ovarian granulosa cells of PCOS patients or healthy controls and identified strong upregulation of miR188-3p. In PCOS, miR-188-3p uncommonly induced, rather than inhibited, its predicted and validated mRNA targets, causing potential altered disease-related pathways.

Gene list for over-representation analysis:

(i) FPR1

(ii) BTG2

(iii) PTAFR

(iv) SPIN3

(v) C3orf38

(vi) SCN9A

(vii) $\mathrm{BACH} 1$

(viii) C3orf52

(ix) METTL16

(x) MCU

(xi) NUP98

(xii) ZNF207

(xiii) SLC2A9

(xiv) CISD1

(xv) NDUFA7

(xvi) TSC22D2

(xvii) CYLD

(xviii) ZNF350

(xix) RAPGEF6

(xx) NUFIP2

(xxi) NNT

(xxii) MCFD2

(xxiii) KLF7

(xxiv) HAS2

\section{Data Availability}

The datasets used and/or analyzed during the current study are available from the corresponding author on reasonable request. 


\section{Conflicts of Interest}

The authors declare that they have no competing interests.

\section{Acknowledgments}

This work is supported by the Youth research project of Shanghai health bureau (Grant No. 20114Y180) (applicant: Beibei Dai).

\section{References}

[1] R. Azziz, L. A. Sanchez, E. S. Knochenhauer et al., "Androgen excess in women: experience with over 1000 consecutive patients," The Journal of Clinical Endocrinology \& Metabolism, vol. 89, no. 2, pp. 453-462, 2004.

[2] G. Bozdag, S. Mumusoglu, D. Zengin, E. Karabulut, and B. O. Yildiz, "The prevalence and phenotypic features of polycystic ovary syndrome: a systematic review and meta-analysis," Human reproduction, vol. 31, no. 12, pp. 2841-2855, 2016.

[3] R. Azziz, E. Carmina, D. Dewailly et al., "Positions statement: criteria for defining polycystic ovary syndrome as a predominantly hyperandrogenic syndrome: an Androgen Excess Society guideline," The Journal of Clinical Endocrinology \& Metabolism, vol. 91, no. 11, pp. 4237-4245, 2006.

[4] Y. Ding, P. He, and Z. Li, "MicroRNA-9119 regulates cell viability of granulosa cells in polycystic ovarian syndrome via mediating Dicer expression," Molecular and Cellular Biochemistry, vol. 465, no. 1-2, pp. 187-197, 2020.

[5] J. Dakshinamoorthy, P. R. Jain, T. Ramamoorthy, R. Ayyappan, and U. Balasundaram, "Association of GWAS identified _INSR_ variants (rs2059807 \& rs1799817) with polycystic ovarian syndrome in Indian women," International Journal of Biological Macromolecules, vol. 144, pp. 663-670, 2020.

[6] S. Mehdinejadiani, F. Amidi, M. Mehdizadeh et al., "Effects of letrozole and clomiphene citrate on Wnt signaling pathway in endometrium of polycystic ovarian syndrome and healthy women†," Biology of Reproduction, vol. 100, no. 3, pp. 641648, 2019.

[7] W. Wang, W. Zhou, S. Wu et al., "Perfluoroalkyl substances exposure and risk of polycystic ovarian syndrome related infertility in Chinese women," Environmental pollution, vol. 247, pp. 824-831, 2019.

[8] H. Shen and Y. Wang, "Activation of TGF- $\beta 1 /$ Smad3 signaling pathway inhibits the development of ovarian follicle in polycystic ovary syndrome by promoting apoptosis of granulosa cells," Journal of Cellular Physiology, vol. 234, no. 7, pp. 11976-11985, 2019.

[9] Q. Yao, Y. Chen, and X. Zhou, "The roles of microRNAs in epigenetic regulation," Current opinion in chemical biology., vol. 51, pp. 11-17, 2019.

[10] A. Valinezhad Orang, R. Safaralizadeh, and M. KazemzadehBavili, "Mechanisms of miRNA-mediated gene regulation from common downregulation to mRNA-specific upregulation," International Journal of Genomics, vol. 2014, Article ID 970607, 15 pages, 2014.

[11] M. Murri, M. Insenser, E. Fernandez-Duran, J. L. San-Millan, and H. F. Escobar-Morreale, "Effects of polycystic ovary syndrome (PCOS), sex hormones, and obesity on circulating miRNA-21, miRNA-27b, miRNA-103, and miRNA-155 expression," The Journal of Clinical Endocrinology \& Metabolism, vol. 98, no. 11, pp. E1835-E1844, 2013.

[12] M. Salimi-Asl, H. Mozdarani, and M. Kadivar, "Up-regulation of miR-21 and 146a expression and increased DNA damage frequency in a mouse model of polycystic ovary syndrome (PCOS)," BioImpacts, vol. 6, no. 2, pp. 85-91, 2016.

[13] M. M. Hossain, M. Cao, Q. Wang et al., "Altered expression of miRNAs in a dihydrotestosterone-induced rat PCOS model," Journal of Ovarian Research, vol. 6, no. 1, 2013.

[14] M. I. Love, W. Huber, and S. Anders, "Moderated estimation of fold change and dispersion for RNA-seq data with DESeq2," Genome Biology, vol. 15, no. 12, 2014.

[15] A. Kucukural, O. Yukselen, D. M. Ozata, M. J. Moore, and M. Garber, "DEBrowser: interactive differential expression analysis and visualization tool for count data," BMC Genomics, vol. 20, no. 1, 2019.

[16] A. Subramanian, P. Tamayo, V. K. Mootha et al., "Gene set enrichment analysis: a knowledge-based approach for interpreting genome-wide expression profiles," Proceedings of the National Academy of Sciences, vol. 102, no. 43, pp. 1554515550, 2005.

[17] H. S. Parker, J. T. Leek, A. V. Favorov et al., "Preserving biological heterogeneity with a permuted surrogate variable analysis for genomics batch correction," Bioinformatics, vol. 30, no. 19, pp. 2757-2763, 2014.

[18] Y. Chen and X. Wang, "miRDB: an online database for prediction of functional microRNA targets," Nucleic Acids Research, vol. 48, no. D1, pp. D127-D131, 2020.

[19] V. Agarwal, G. W. Bell, J. W. Nam, and D. P. Bartel, "Predicting effective microRNA target sites in mammalian mRNAs," eLife, vol. 4, 2015.

[20] M. D. Paraskevopoulou, G. Georgakilas, N. Kostoulas et al., "DIANA-microT web server v5.0: service integration into miRNA functional analysis workflows," Nucleic acids research, vol. 41, no. W1, pp. W169-W173, 2013.

[21] H. Y. Huang, Y. C. Lin, J. Li et al., "miRTarBase 2020: updates to the experimentally validated microRNA-target interaction database," Nucleic Acids Research, vol. 48, no. D1, pp. D148D154, 2020.

[22] M. V. Kuleshov, M. R. Jones, A. D. Rouillard et al., "Enrichr: a comprehensive gene set enrichment analysis web server 2016 update," Nucleic Acids Research, vol. 44, no. W1, pp. W90W97, 2016.

[23] T. T. Piltonen, J. Chen, D. W. Erikson et al., "Mesenchymal stem/progenitors and other endometrial cell types from women with polycystic ovary syndrome (PCOS) display inflammatory and oncogenic potential," The Journal of Clinical Endocrinology \& Metabolism, vol. 98, no. 9, pp. 37653775, 2013.

[24] M. Koziczak-Holbro, C. Joyce, A. Glück, B. Kinzel, M. Müller, and H. Gram, "Solving the IRAK-4 enigma: application of kinase-dead knock-in mice," in Sparking Signals, vol. 3 of Ernst Schering Foundation Symposium Proceedings, pp. 263-282, Springer, Berlin, Heidelberg, 2008.

[25] Z. Madak-Erdogan, M. Lupien, F. Stossi, M. Brown, and B. S. Katzenellenbogen, "Genomic collaboration of estrogen receptor alpha and extracellular signal-regulated kinase 2 in regulating gene and proliferation programs," Molecular and Cellular Biology, vol. 31, no. 1, pp. 226-236, 2011.

[26] D. N. Slenter, M. Kutmon, K. Hanspers et al., "WikiPathways: a multifaceted pathway database bridging metabolomics to 
other omics research," Nucleic Acids Research, vol. 46, no. D1, pp. D661-D667, 2018.

[27] C. F. Schaefer, K. Anthony, S. Krupa et al., "PID: the Pathway Interaction Database," Nucleic Acids Research, vol. 37, supplement 1, pp. D674-D679, 2009.

[28] B. Chen, P. Xu, J. Wang, and C. Zhang, "The role of miRNA in polycystic ovary syndrome (PCOS)," Gene, vol. 706, pp. 91-96, 2019.

[29] L. W. Roth, B. McCallie, R. Alvero, W. B. Schoolcraft, D. Minjarez, and M. G. Katz-Jaffe, "Altered microRNA and gene expression in the follicular fluid of women with polycystic ovary syndrome," Journal of Assisted Reproduction and Genetics, vol. 31, no. 3, pp. 355-362, 2014.

[30] A. V. Sirotkin, M. Laukova, D. Ovcharenko, P. Brenaut, and M. Mlyncek, "Identification of microRNAs controlling human ovarian cell proliferation and apoptosis," Journal of Cellular Physiology, vol. 223, no. 1, pp. 49-56, 2010.

[31] Y. H. Chen, S. Heneidi, J. M. Lee et al., "miRNA-93 inhibits GLUT4 and is overexpressed in adipose tissue of polycystic ovary syndrome patients and women with insulin resistance," Diabetes, vol. 62, no. 7, pp. 2278-2286, 2013.

[32] M. Yin, X. Wang, G. Yao et al., "Transactivation of microRNA320 by microRNA-383 regulates granulosa cell functions by targeting E2F1 and SF-1 proteins," Journal of Biological Chemistry, vol. 289, no. 26, pp. 18239-18257, 2014.

[33] Q. Sang, Z. Yao, H. Wang et al., "Identification of microRNAs in human follicular fluid: characterization of microRNAs that govern steroidogenesis in vitro and are associated with polycystic ovary syndrome in vivo," The Journal of Clinical Endocrinology \& Metabolism, vol. 98, no. 7, pp. 3068-3079, 2013.

[34] B. Xu, Y. W. Zhang, X. H. Tong, and Y. S. Liu, "Characterization of microRNA profile in human cumulus granulosa cells: identification of microRNAs that regulate Notch signaling and are associated with PCOS," Molecular and Cellular Endocrinology, vol. 404, pp. 26-36, 2015.

[35] L. Shi, S. Liu, W. Zhao, and J. Shi, "miR-483-5p and miR486-5p are down-regulated in cumulus cells of metaphase II oocytes from women with polycystic ovary syndrome," Reproductive BioMedicine Online, vol. 31, no. 4, pp. 565572, 2015.

[36] M. Pichler, V. Stiegelbauer, P. Vychytilova-Faltejskova et al., "Genome-wide miRNA analysis identifies miR-188-3p as a novel prognostic marker and molecular factor involved in colorectal carcinogenesis," Clinical Cancer Research, vol. 23, no. 5, pp. 1323-1333, 2017.

[37] W. Y. Song, H. Meng, X. G. Wang et al., "Reduced microRNA188-3p expression contributes to apoptosis of spermatogenic cells in patients with azoospermia," Cell proliferation, vol. 50, no. 1, article e12297, 2017.

[38] K. Wang, C. Y. Liu, L. Y. Zhou et al., "APF lncRNA regulates autophagy and myocardial infarction by targeting miR-1883p," Nature Communications, vol. 6, no. 1, 2015.

[39] X. F. Zhang, Y. Yang, X. Y. Yang, and Q. Tong, "MiR-188-3p upregulation results in the inhibition of macrophage proinflammatory activities and atherosclerosis in ApoE-deficient mice," Thrombosis Research, vol. 171, pp. 55-61, 2018.

[40] S. Vasudevan, Y. Tong, and J. A. Steitz, "Switching from repression to activation: microRNAs can up-regulate translation," Science, vol. 318, no. 5858, pp. 1931-1934, 2007.
[41] C. L. Jopling, S. Schutz, and P. Sarnow, "Position-dependent function for a tandem microRNA miR-122-binding site located in the hepatitis C virus RNA genome," Cell Host \& Microbe, vol. 4, no. 1, pp. 77-85, 2008.

[42] Y. Li, T. Masaki, D. Yamane, D. R. McGivern, and S. M. Lemon, "Competing and noncompeting activities of miR-122 and the 5 ' exonuclease $\mathrm{Xrn} 1$ in regulation of hepatitis $\mathrm{C}$ virus replication," Proceedings of the National Academy of Sciences, vol. 110, no. 5, pp. 1881-1886, 2013.

[43] T. Shimakami, D. Yamane, R. K. Jangra et al., "Stabilization of hepatitis C virus RNA by an Ago2-miR-122 complex," Proceedings of the National Academy of Sciences, vol. 109, no. 3, pp. 941-946, 2012.

[44] U. A. Orom, F. C. Nielsen, and A. H. Lund, "MicroRNA-10a binds the $5^{\prime}$ UTR of ribosomal protein mRNAs and enhances their translation," Molecular Cell, vol. 30, no. 4, pp. 460-471, 2008.

[45] K. M. Hoeger, "Obesity and lifestyle management in polycystic ovary syndrome," Clinical Obstetrics and Gynecology, vol. 50, no. 1, pp. 277-294, 2007.

[46] R. S. Legro, "Obesity and PCOS: implications for diagnosis and treatment," Seminars in Reproductive Medicine, vol. 30, no. 6, pp. 496-506, 2012.

[47] X. L. Zeng, Y. F. Zhang, Q. Tian, Y. Xue, and R. F. An, “Effects of metformin on pregnancy outcomes in women with polycystic ovary syndrome: a meta-analysis," Medicine, vol. 95, no. 36, article e4526, 2016.

[48] M. Liu, J. Gao, Y. Zhang et al., "Serum levels of TSP-1, NF- $\kappa$ B and TGF- $\beta 1$ in polycystic ovarian syndrome (PCOS) patients in northern China suggest PCOS is associated with chronic inflammation," Clinical Endocrinology, vol. 83, no. 6, pp. 913-922, 2015.

[49] M. Rostamtabar, S. Esmaeilzadeh, M. Tourani et al., "Pathophysiological roles of chronic low-grade inflammation mediators in polycystic ovary syndrome," Journal of Cellular Physiology, vol. 236, no. 2, pp. 824-838, 2021.

[50] Y. Zhao, C. Zhang, Y. Huang et al., "Up-regulated expression of WNT5a increases inflammation and oxidative stress via $\mathrm{PI} 3 \mathrm{~K} / \mathrm{AKT} / \mathrm{NF}-\kappa \mathrm{B}$ signaling in the granulosa cells of PCOS patients," The Journal of Clinical Endocrinology \& Metabolism, vol. 100, no. 1, pp. 201-211, 2015.

[51] T. Zuo, M. Zhu, W. Xu, Z. Wang, and H. Song, "Iridoids with genipin stem nucleus inhibit lipopolysaccharide-induced inflammation and oxidative stress by blocking the NF- $\kappa \mathrm{B}$ pathway in polycystic ovary syndrome," Cellular Physiology and Biochemistry, vol. 43, no. 5, pp. 1855-1865, 2017. 